\title{
Potential field continuation: a comparative analysis of three different types of software
}

\author{
Marcello Ciminale and Mariano Loddo \\ Dipartimento di Geologia e Geofisica, Università di Bari, Italy
}

\begin{abstract}
DARING.F is a new Fortran77 computer program which has been developed to perform the continuation of potential field data between arbitrary surfaces. The implemented equivalent source algorithm inverts a system and that of two computer programs matrix. A comparative analysis between the performance of this software thors is carried out As a programs (named UPWARD.F and UPNEW.F) previously written by the same auhighest level of accuracy of transformed dalysis, some useful and important suggestions on how to obtain the are given.
\end{abstract}

Key words potential fields - equivalent sources sparse matrix - computer programs

\section{Introduction}

When magnetic or gravity data are observed on an arbitrary surface characterized by a high relief or by abrupt variations in altitude, some spurious anomalies or significant distortions may be generated in the signal. If the aim of the geophysical investigation is to keep all the information connected to shallow and deep sources, only the reduction of the topographic interferences is necessary and this is obtained by upward continuing to a horizontal plane located as close as possible to the observation surface. On the other hand, if an improved

Mailing address: Prof. Marcello Ciminale, Dipartimento di Geologia e Geofisica, Università di Bari, Campus Universitario, Via E. Orabona 4, 70125 Bari, Italy; e-mail: marci@geo.uniba.it resolution of the potential field data associated with the more extended and deeper bodies is required, a reduction of the masking effect of the shallower sources also becomes necessary and thus, an upward continuation to a more distant horizontal plane is suitable.

In their first paper (Ciminale and Loddo, 1989), the authors proposed a computer program based on an equivalent source algorithm and on the solution of the consequent Fredholm integral equation of the second kind. This approach was successful in solving the problem very well but, later on, some serious limitations were observed when continuing between close surfaces. The limitations were subsequently overcome by introducing a more refined approximation to the equivalent source distribution (Ray and Hansen, 1992; Ciminale and Loddo, 1992).

Recently, a completely new software package, DARING.F, has been developed; the equivalent source technique is still applied but now a system of linear equations is resolved by an inversion method which adopts sparse matrix. 
In this paper, the mathematical background, the algorithm and the structure of DARING.F are illustrated first. Its performance is then compared with those of the two previously elaborated softwares. Finally, some useful and important suggestions about their most appropriate use (especially when large matrices are processed and/or close surfaces are considered) are proposed.

\section{Mathematical background}

The equivalent source technique was first proposed by Dampney (1969). Since then, it has been widely used by many authors (e.g., Emilia, 1973; Bhattacharyya and Chan, 1977; Hansen and Miyazaki, 1984; Ciminale and Loddo, 1989; Graber Brunner et al., 1991; Mendonca and Silva, 1994) for transforming potential field data (continuation, first and second vertical derivative, pseudogravity, reduction to the pole, etc.). In this paper, the problem of continuation is considered and is directly approached in discrete form.

Thus, let $S$ be an arbitrary discrete surface on which potential field measurements are collected on a regular $(N \times M)$ grid. By using the equivalent source method, it is possible to define a layer of given thickness, formed by $N \times M$ contiguous equivalent source cells and placed at a constant depth from $S$.

The following equation can be written:

$$
\alpha_{i j}=\sum_{k=1}^{N} \sum_{l=1}^{M} W_{k l}^{i j} \vartheta_{k l}
$$

or in matrix form

$$
\boldsymbol{A}=\boldsymbol{B} \boldsymbol{C}
$$

where

$$
i=1,2, \ldots \ldots, N ; j=1,2, \ldots \ldots \ldots, M
$$

and $\alpha_{i j}$, element of $\boldsymbol{A}$, is the gravitational or magnetic effect measured on $S$ at the station point $(i, j) ; W_{k l}^{i j}$ is the entry of the $(N \times M)^{2}$ matrix $\boldsymbol{B}$ which at $(i, j)$ represents the potential field effect, due to the equivalent cell $(k, l)$ having a unitary density (gravity case) or unitary magnetization (magnetic case); $\vartheta_{k l}$, which belongs to vector $\boldsymbol{C}$, is the unknown equivalent value (density or intensity of magnetization) to attribute to the cell centered at the point $(k, l)$. Equation (2.1) describes a linear inverse problem; it defines a mathematical relationship between observed data and theoretical sources. To solve it means determining a distribution of theoretical sources whose field values on $S$ are exactly equal to the measured data. Therefore, the potential field generated by these sources on a different surface $S^{\prime}$ represents the continuation of the experimental data to this new surface $S^{\prime}$.

\section{Numerical procedure}

To solve eq. (2.1), it is first necessary to fix the geometry and location of the equivalent layer and to determine the $W_{k l}^{i j}$ entries. Theoretically, the equivalent layer does not have to be at a constant depth from $S$ although, assuming equivalent cells at different depths will degrade the method's efficiency and make it less operational. Thus, since data on $S$ are distributed on a regular grid (square or rectangle meshes) and the topographic level at the nodes of this grid is also known, it is possible to define a discrete layer. Such a layer is formed by $N \times M$ prisms, having a given thickness and top sides placed at a constant depth $\mathrm{cl}$ from the level altitude of the grid points to which they are connected. Once the equivalent layer has been defined, the computation of the coefficients $W_{k l}^{i j}$ is executed by simple routines based on the formulas written by Nagy (1966) and Bhattacharyya (1964) for the gravity and the magnetic case respectively.

Equation (2.1) represents a system of $N$ times $M$ linear equations and $N$ times $M$ unknowns; its direct solution is not mathematically complex but, if large data sets are handled, long computing time and very large RAM memory is required and even a computer system with a medium-high performance may fail to execute the computation because of the great dimension of matrix $\boldsymbol{B}$. These serious limitations are well known to those who are fa- 
miliar with linear inverse problems and in general, iterative methods are applied to obtain the best approximate solution. In this research paper a different method for solving eq. (2.1) is proposed: the potential field effects decrease in accordance with the inverse (square or cubic) distance law. Therefore, contributions of cells $(k, l)$ which are far away from the measuring point $(i, j)$, could be considered negligible and set equal to zero. This physically correct approximation allows the handling of matrix $\boldsymbol{B}$ as a sparse matrix; its processing presents significant advantages both in speed and in storage requirements. DARING.F computes, for each point $(i, j)$ all the $N \times M W_{k l}^{i j}$ contributions; when their result is lower than a given percentage cut, of the highest value (connected to the closest cell) they are set equal to zero. The package of routines TDRV for a sparse nonsymmetric matrix (Eisenstat et al., 1977) is then applied and its usage saves both time and storage memory. This results in computations with very good approximation. The fact that very low values of $c u t(\cong 0.1 \%)$ are suitable to obtain accurate data will be shown in the next section.

Once the equivalent source distribution is completely known (location, geometry and equivalent value of the physical parameter), the calculation of the potential field on an arbitrary surface $S^{\prime}$ external to the equivalent layer becomes a straightforward procedure using the routines based on the formula given by Nagy (1966) or Bhattacharyya (1964).

The program DARING.F is divided into four parts:

a) Input: data and parameters reading.

b) Preinversion: geometry and location of the equivalent layer and $W_{k l}^{i j}$ entry computation. tation.

c) Inversion: equivalent source compu-

d) Output: continuation.

In part (a), input data and parameters such as potential field and topographic data, surface or level of continuation, value of the clearance $c l$, value of the parameter cut, output filename are introduced. Part (b) determines the geometry and location of the equivalent prism-shaped cells and the values of $W_{k l}^{i j}$. Equation (2.1) is inverted in part (c) by using the package TDRV; matrix $\boldsymbol{B}$ is handled as a sparse matrix if $c u t \neq 0$ and the equivalent values $\vartheta_{k l}$ are obtained. Finally, the continuation is executed in part (d) following the straightforward procedure illustrated in the previous paragraph.

At present, this program runs on a Digital Alpha 300 machine provided with a RAM memory of $64 \mathrm{MB}$, a hard disk of $3 \mathrm{~GB}$ and a clock frequency of $175 \mathrm{Mhz}$.

\section{Comparative analysis}

In the first paper (Ciminale and Loddo, 1989) the upward continuation of potential field data was performed with the program UPWARD.F based on the algorithm suggested by Bhattacharyya and Chan (1977). Tests included in that article clearly showed the program's ability to reduce significant topographic interference with the signal. Some time later however, it was noted that some problems arose when continuation was carried out between close surfaces (e.g., less than 1 grid unit away). Thanks to some suggestions proposed by Ray and Hansen (1992), some routines were amended by introducing a more refined approximation to the equivalent source distribution (Ciminale and Loddo, 1992) and the upgraded version UPNEW.F is effective and accurate in this case as well.

In this section, a comparative analysis among UPWARD.F, UPNEW.F and DARING.F (hereafter referred to as UPW, UPN, DAR) is carried out to examine the effectiveness of these programs in terms of data accuracy, time consumption and storage memory requirement both when matrices with different dimensions are processed and when very close surfaces are considered.

Figure 1 shows the processed high topographic relief. It is a $33 \times 33$ regular grid having a station spacing equal to $1000 \mathrm{~m}$; the top value is $3160 \mathrm{~m}$. Figure $2 \mathrm{a}, \mathrm{b}$ shows the total magnetic field and the gravity effect respectively, both computed on the topographic relief and resulting from the five prismatic bodies outlined in fig. 1. The total magnetic field has been calculated by assuming a normal mag- 


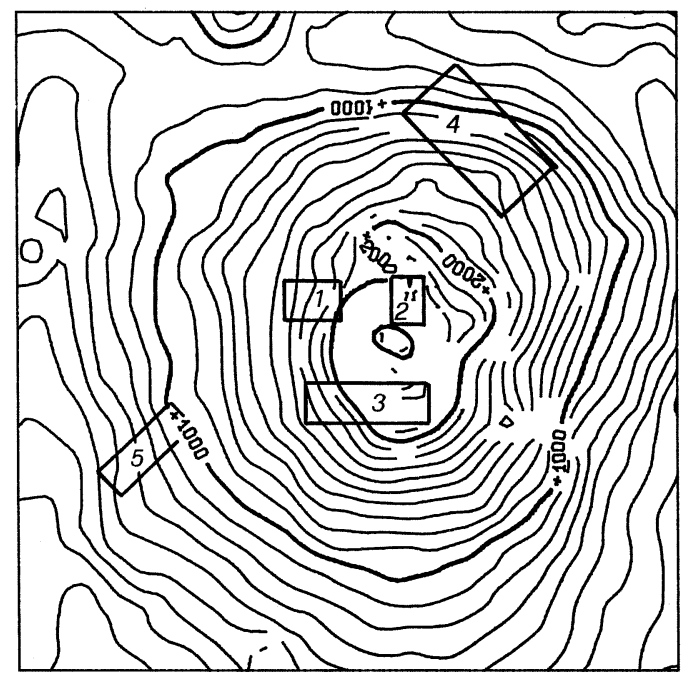

Fig. 1. Topographic model. Dimensions of the area are $32 \mathrm{~km} \times 32 \mathrm{~km}$. Contour interval is $100 \mathrm{~m}$. The outlines of the five synthetic source bodies are also shown. netic field having an inclination $I_{0}=65^{\circ} \mathrm{N}$ and a declination $D_{0}=20^{\circ} \mathrm{E}$. The parameters which identify the prisms are reported in table I. The magnetic field was upward continued onto a horizontal plane whose level altitude, $3200 \mathrm{~m}$, is only $40 \mathrm{~m}$ from the highest elevation of the observation surface: UPW, UPN and DAR were used. In fig. 3a-d, results of these transformations are shown; the strong distortion which appears in fig. 3a (UPW application), exactly in connection with the location of the top of the mountain, is highly attenuated in fig. 3b (UPN application) and completely vanishes in fig. 3c (DAR application). These results, compared with the total field which is the result of the same five prismatic bodies computed at a horizontal plane $3200 \mathrm{~m}$ above mean sea level (fig. 4), outline the high performance of DAR. Fig. 3c has been computed with $c u t=0 \%$ that is, all $W_{k l}^{i j}$ coefficients $(1,185,921)$ were used for inverting the system and matrix $\boldsymbol{B}$ was processed as a dense matrix. The CPU time for this computation was 294 s. Figure 3d
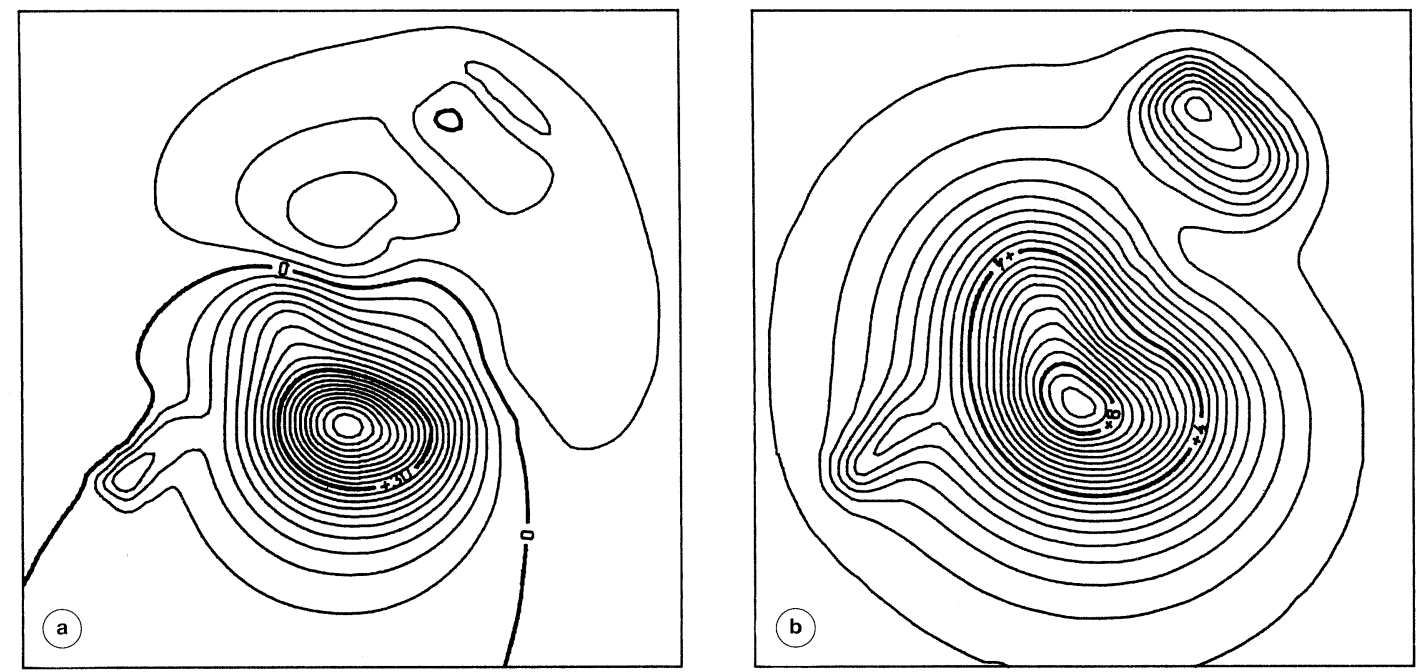

Fig. 2a,b. a) Total magnetic field, resulting from the five prismatic bodies, computed on the topographic surface shown in fig. 1. Contour interval is $3 \mathrm{nT}$. b) Gravity effect, resulting from the five prismatic bodies, computed on the topographic surface shown in fig. 1 . Contour interval is $0.4 \mathrm{mgal}$. 
Table I. Characters of prisms; $N=$ prism numeration; $T=$ depth of the top; $B=$ depth of the bottom; $M=$ intensity of magnetization; $D_{\epsilon}=$ characteristic declination; $D_{i}=$ characteristic inclination; $\sigma=$ contrast of
density.

\begin{tabular}{ccccccc}
\hline$N$ & $T(\mathrm{~km})$ & $B \mathrm{~km})$ & $M(\mathrm{~A} / \mathrm{m})$ & $D_{e}$ & $D_{i}$ & $\sigma\left(\mathrm{g} / \mathrm{cm}^{3}\right)$ \\
\hline 1 & 2.0 & 7.0 & 1.0 & $45^{\circ} \mathrm{E}$ & $30^{\circ} \mathrm{N}$ & 0.8 \\
2 & 3.0 & 7.0 & 2.0 & $5^{\circ} \mathrm{E}$ & $72^{\circ} \mathrm{N}$ & -1.4 \\
3 & 1.5 & 6.0 & 1.0 & $10^{\circ} \mathrm{E}$ & $60^{\circ} \mathrm{N}$ & 0.8 \\
4 & 0.2 & 0.35 & 0.3 & $10^{\circ} \mathrm{E}$ & $60^{\circ} \mathrm{N}$ & 1.1 \\
5 & 0.2 & 0.35 & 0.3 & $10^{\circ} \mathrm{E}$ & $60^{\circ} \mathrm{N}$ & 0.7 \\
\hline
\end{tabular}
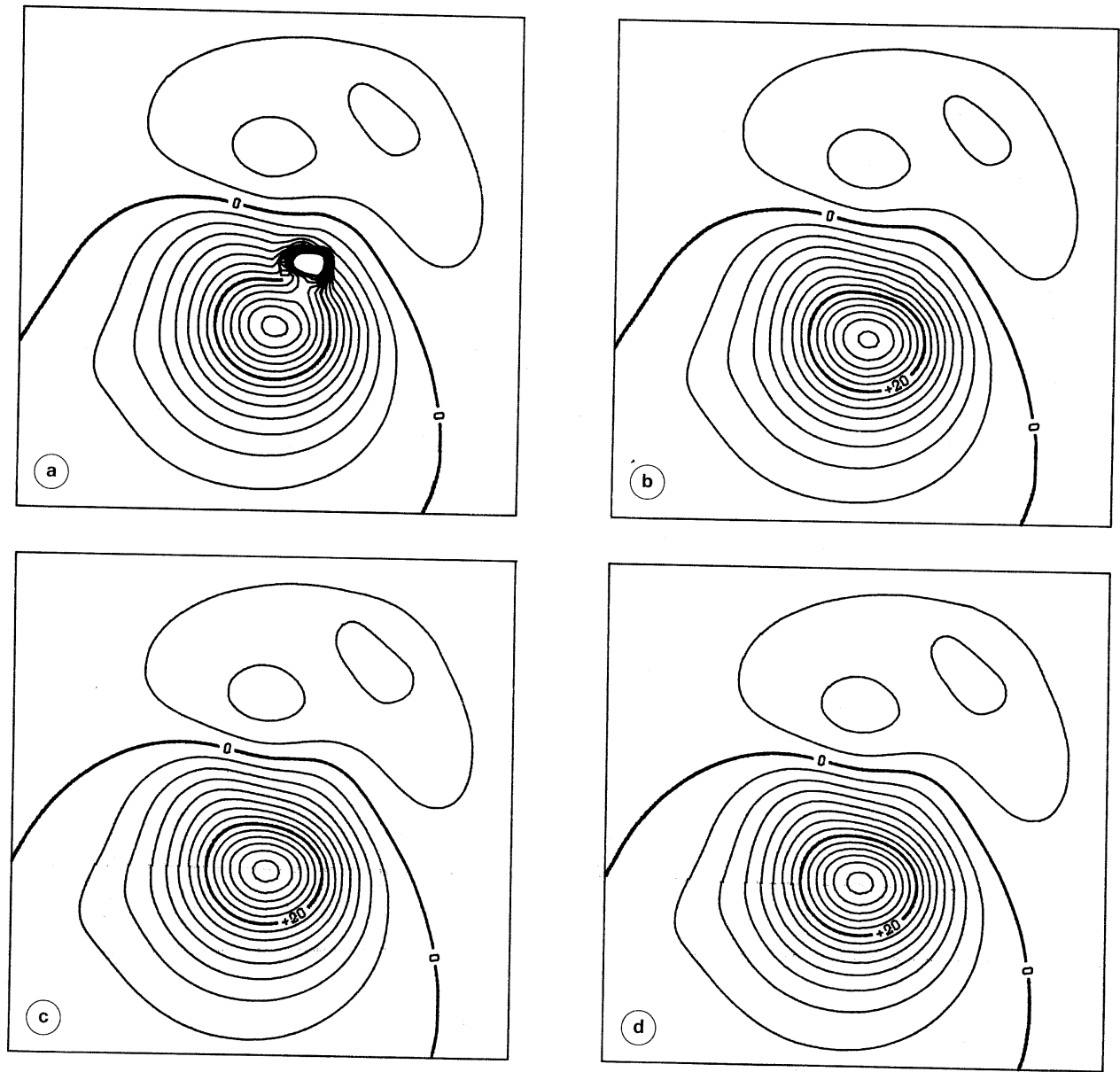

Fig. 3a-d. Upward continuation to a level of $3200 \mathrm{~m}$ of the total magnetic field shown in fig. 2a. Contour interval is $2.5 \mathrm{nT}$. a) UPWARD.F application; b) UPNEW.F application; c) DARING.F application, $c u t=0.0 \%$;
d) DARING.F application, cut $=0.1 \%$. 


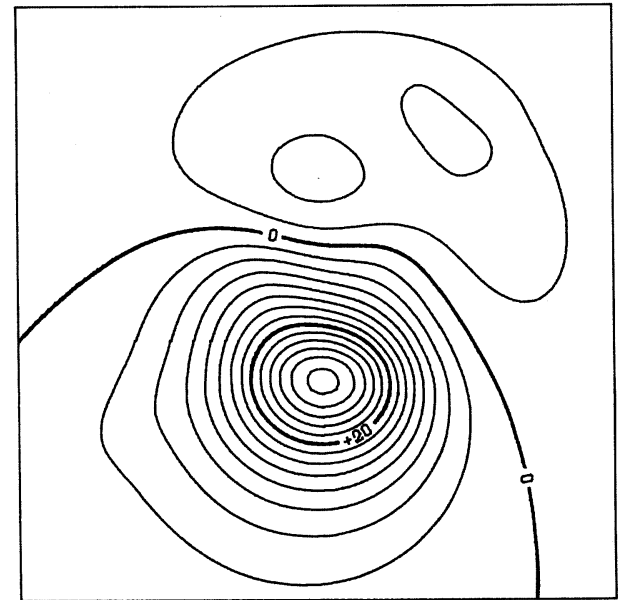

Fig. 4. Total magnetic field, resulting from the five prismatic bodies outlined in fig. 1, computed at a level of $3200 \mathrm{~m}$. Contour interval is $2.5 \mathrm{nT}$.

has been obtained by putting $c u t=0.1 \%$. The matching of this map with fig. $3 \mathrm{c}$ and fig. 4 is very good; only 738,407 entries were used (a reduction of $38 \%$ ) and a CPU time of $213 \mathrm{~s}$ was necessary (about $27 \%$ time saving).

The upward continuation to the altitude of $3200 \mathrm{~m}$ of the gravity signal shown in fig. $2 \mathrm{~b}$ is given in fig. 5a-c. In this case the best result is still obtained by running program DAR (fig. 5c): no high frequency distortions connected to topographic relief are present and the matching with the signal generated by the prismatic bodies on the $3200 \mathrm{~m}$ horizontal plane (fig. 6) is highly satisfactory.

If the continuation is carried out to a higher level, for instance $4800 \mathrm{~m}$, the three output data become comparable (fig. 7a-c): no more instabilities are present when applying UPW and UPN. The behaviour, shape and amplitude

Fig. 5a-c. Upward continuation to a level of $3200 \mathrm{~m}$ of the gravity effect shown in fig. $2 b$. Contour interval is 0.4 mgal. a) UPWARD.F application; b) UPNEW.F application; c) DARING.F application, $c u t=0.0 \%$.
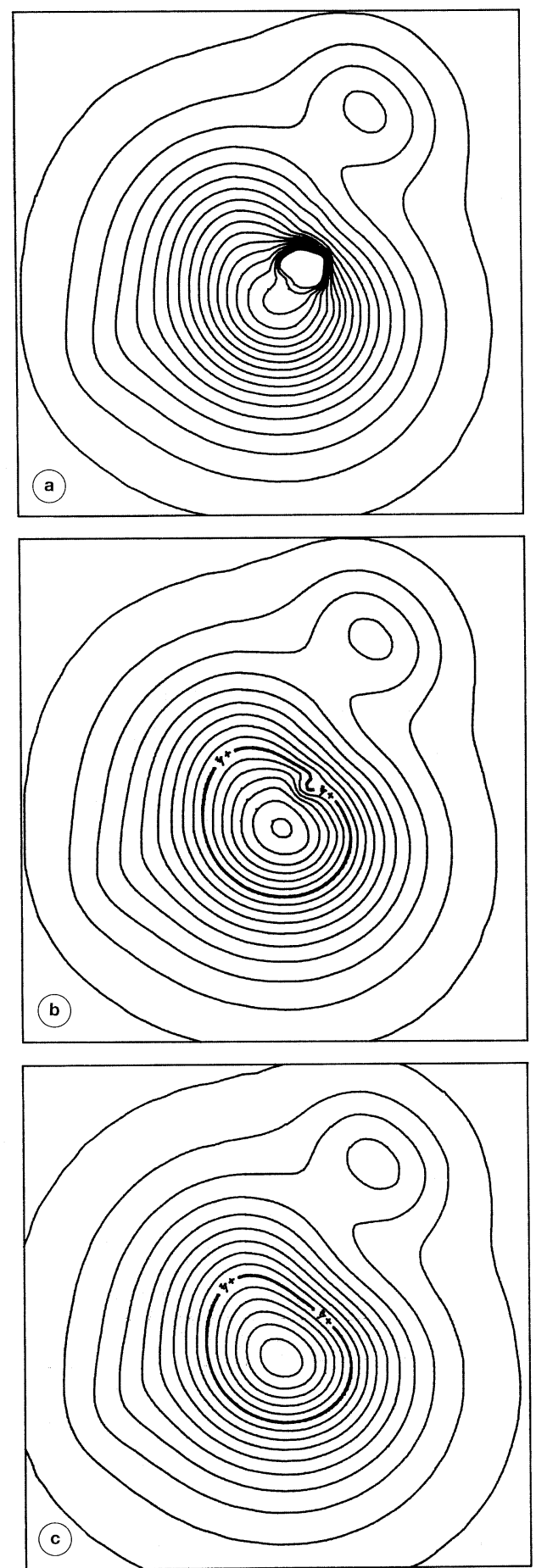


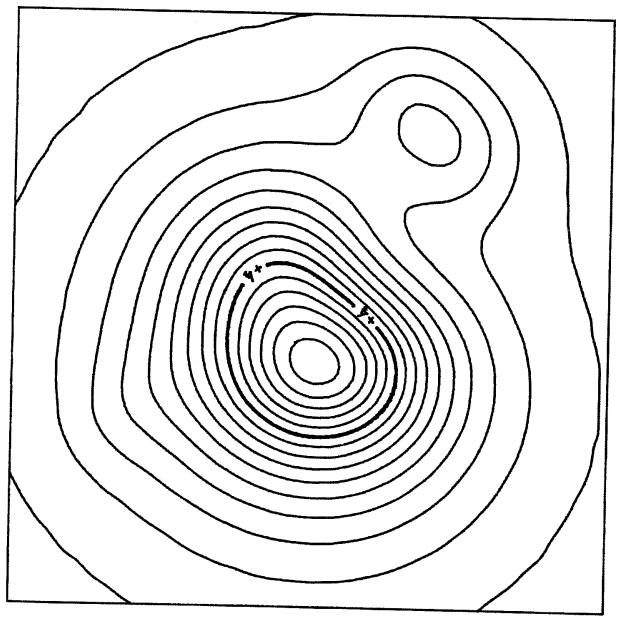

Fig. 6. Gravity effect, resulting from the five prismatic bodies outlined in fig. 1, computed at a level of $3200 \mathrm{~m}$. Contour interval is $0.4 \mathrm{mgal}$.

of the three signals are quite similar. The best result is still obtained by DAR (compare with fig. 8). However, if the time consumption is taken into consideration, then it must be noted that DAR took $294 \mathrm{~s}$ to execute the computation while UPW and UPN required only $13 \mathrm{~s}$ : a remarkable time saving.

The last test, which is illustrated below, regards real gravity data collected on a rugged topography (Sicily, Italy) whose top level was $2079 \mathrm{~m}$. The regular grid $(44 \times 44)$ of Bouguer anomalies also contains some gaps. This is due to the absence of measurements because some areas were inaccessible (i.e. woods, sea, etc.). The Bouguer anomaly map is shown in fig. 9. The input/output procedures of UPW, UPN and DAR require a full grid. At present, only program DAR can transform a non-complete grid because it has been provided with a package of routines which temporarily fills the

Fig. 7a-c. As in fig. 5a-c but the level of continuation is $4800 \mathrm{~m}$. Contour interval is $0.25 \mathrm{mgal}$; a) UPWARD.F application; b) UPNEW.F application; c) DARING.F application, cut $=0.0 \%$.
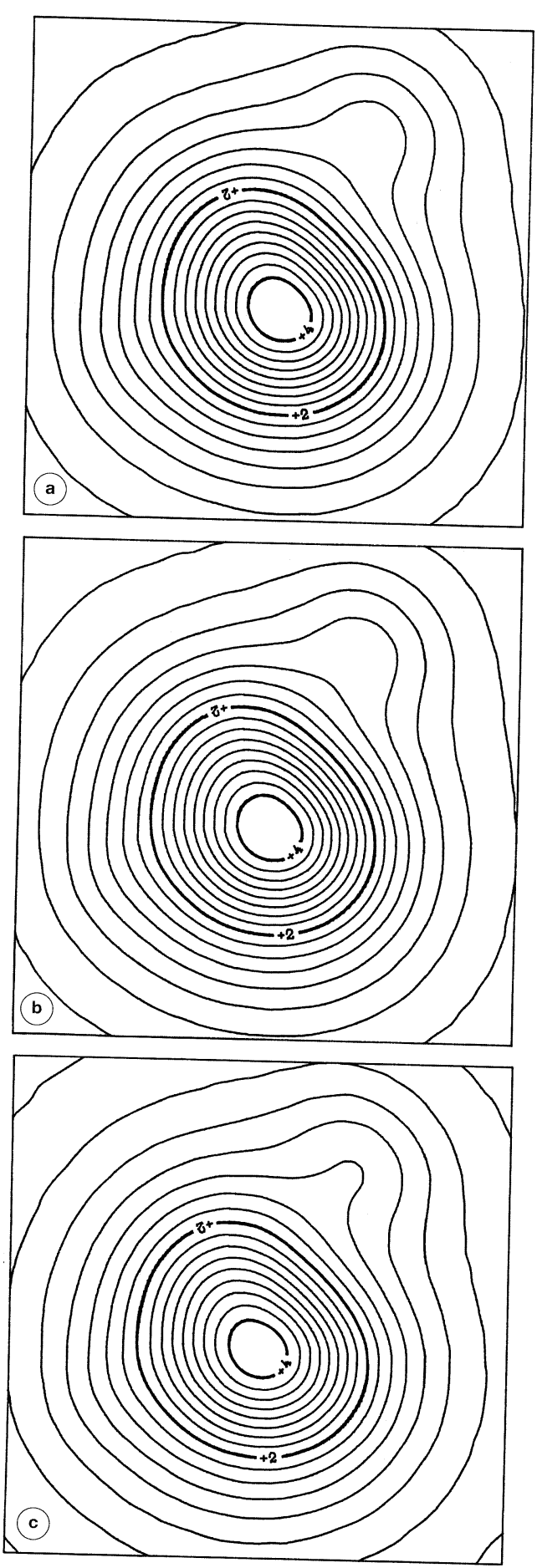


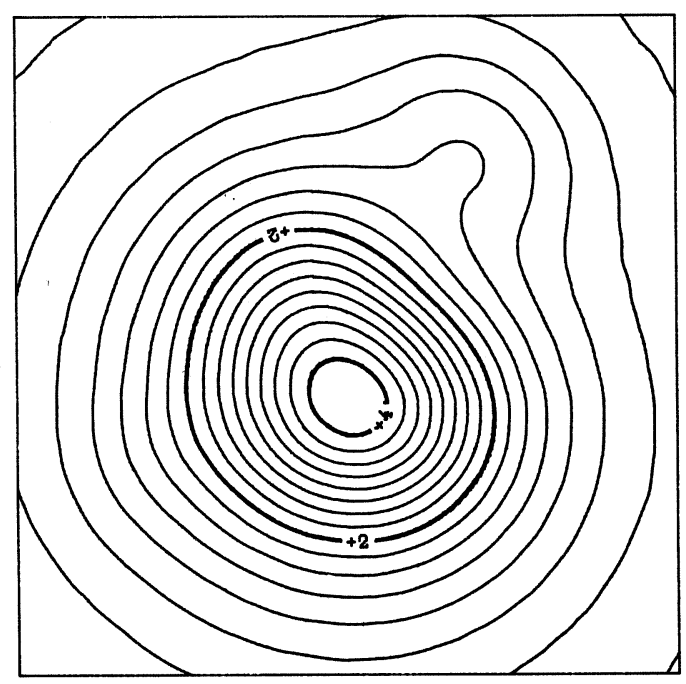

Fig. 8. As in fig. 6 but the level of computation is $4800 \mathrm{~m}$. Contour interval is $0.25 \mathrm{mgal}$.

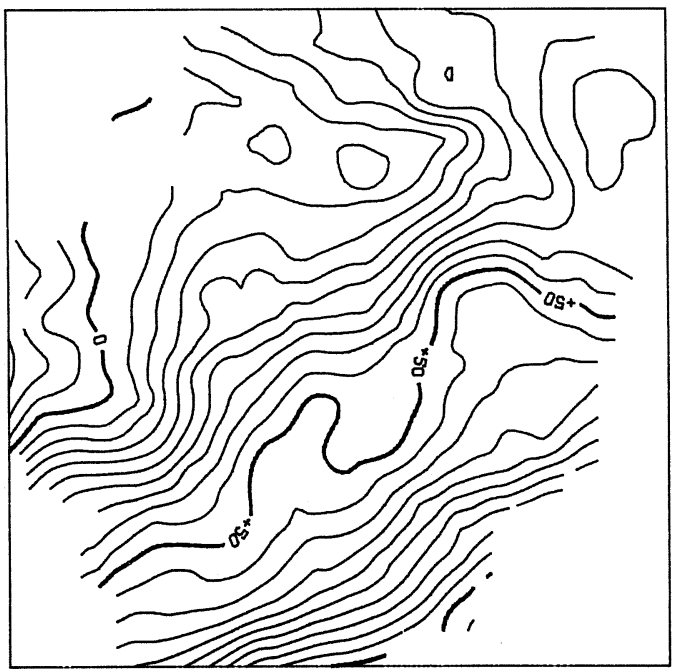

Fig. 9. Experimental Bouguer anomalies collected in Sicily (Italy). Dimensions of the area are 43 $\mathrm{km} \times 43 \mathrm{~km}$. Contour interval is $5 \mathrm{mgal}$. gaps with a suitable method for extrapolating (Gibert and Galdeano, 1985) derived from Burg's Maximum Entropy Spectral Analysis algorithm (Burg, 1975). The Bouguer map was upward continued to a level of $2100 \mathrm{~m}$ (fig. 10a). This result was obtained by handling matrix $\boldsymbol{B}$ as a dense matrix: all its entries $3,748,096$ in total - were processed. In this case the CPU time required was $700 \mathrm{~s}$; putting cut $=0.1 \%$ only 588,599 coefficients are considered and the CPU time is reduced to $297 \mathrm{~s}$ representing a substantial storage and time saving without penalizing accuracy (fig. 10b).

\section{Comments and conclusions}

In the previous section synthetic and real data were transformed by running programs UPW, UPN and DAR. DAR has proven not only to have the highest level of accuracy in data continuation, but also to be able to execute the upward continuation between surfaces which are very close or even tangent. This important option is due to the fact that the equivalent sources are placed below the observation surface while the algorithm included in UPW and UPN requires that the equivalent layer and the observation surface coincide. Locating the equivalent sources below the observation surface, also allows downward continuation. In this transformation, great care must be taken when choosing the continuation level for it must not go beyond the clearance level which runs between the observation surface and the equivalent source layer. Finally, DAR is the only program, at present, which can process non-dense grids of data (see the test with real data). All these observations represent the strongest points of the DAR program.

DAR's weakest points are the long computing time and the very large RAM memory needed for its performance. The CPU time (294 s) required by DAR to transform a $33 \times 33$ matrix correctly could still be considered acceptable. However, if compared to the fraction of time required by UPN to carry out the same transformation (13 s), it appears obvious that a great loss in speed is suffered. The dimension of matrix $\boldsymbol{B}$ is $(N \times M)^{2}$ and it will magnify if a 

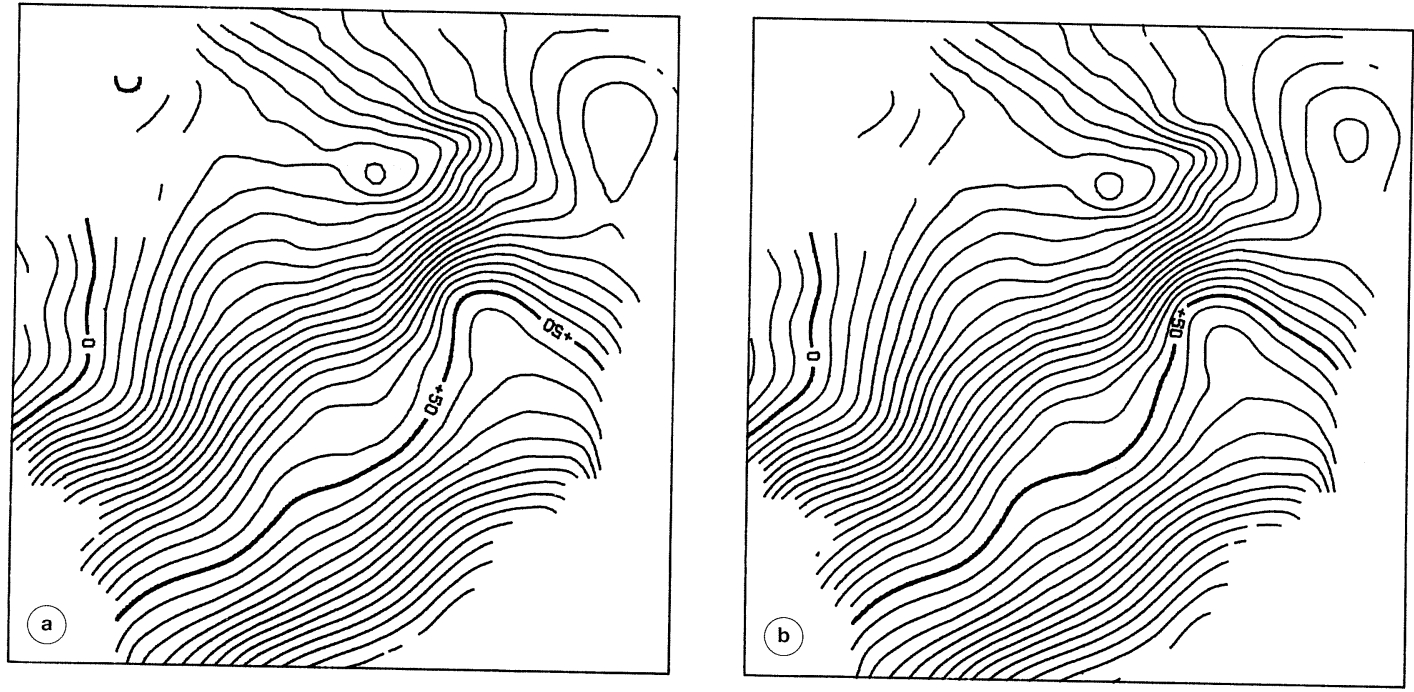

Fig. 10a,b. Data of fig. 9 upward continued to a level of $2100 \mathrm{~m}$ by running DARING.F. Contour interval is 2.5 mgal; a) $c u t=0.0 \%$; b) $c u t=0.1 \%$.

large data set is processed. Hence the dimension of $\boldsymbol{B}$, could collide with the computer memory limitation. Fortunately, the introduction of an appropriate value of cut parameter can exclude all $W_{k l}^{i j}$ entries which do not give significant contributions thereby reducing the dimension of $\boldsymbol{B}$ to lower problematic values. These problems however, do not appear in the programs UPW and UPN. Another crucial factor which weakens DAR's performance is the correct determination of the clearance separating the observation surface from the equivalent layer. A direct general rule fixing the best location of the equivalent layer below the measuring surface does not exist. In fact, if the source depth is either too shallow with respect to the station spacing or too deep, then aliasing phenomena or high frequency noise will occur when projecting potential field anomalies onto a new surface. Dampney (1969) observes in a semiempirical way that the determinations of the $W_{k l}^{i j}$ entries become reliable if the value of the clearance lies within the interval $(2.5 \Delta$, $6 \Delta)$ where $\Delta$ is the station spacing. More recently, Xia and Sprowl (1991) have mathematically determined the optimum depth to the source ensemble by adopting a rule which maximizes the smoothness of the calculated anomaly between data points. On the basis of their examples, the best solution is obtained by placing the equivalent layer at a depth lying within the interval $(1 \Delta, 2 \Delta)$. At present, the authors are not able to offer suggestions for an efficient method to determine the optimum depth. However, by following some considerations made by Xia and Sprowl (1991) it has been empirically found that the transformations illustrated in the previous section are obtained by placing the equivalent layer at a depth of $2.5 \Delta, 1.5 \Delta$ and $0.86 \Delta$ for the magnetic, gravimetric and real case, respectively. Moreover, it has been observed that no significant variations in the output data are introduced when changing the thickness $(t k s)$ of the equivalent cells and thus it can be introduced in DARING.F as a given parameter $(t k s=0.5,1.0$ or 1.5 for instance).

In conclusion, this comparative analysis among UPW, UPN and DAR but, in actual fact, between UPNEW.F and DARING.F, suggests that if a very large matrix of data must be processed by continuing between surfaces which are more than about 1 grid unit away, program UPNEW.F yields very good results 
and saves considerable time. However, if the grid of data is not very large and the continuation to surfaces which are very close is required, a very high level of accuracy of data output is given by correctly applying (determination of the optimum depth) program DARING.F.

\section{Acknowledgements}

Financial support for this research was provided by MURST $(40 \%, 95)$ and CNR (Contract No. 96.363.02)

\section{REFERENCES}

BHATTACHARYYA, B.K. (1964): Magnetic anomalies due to prism-shaped bodies with arbitrary polarization, Geophysics, 29, 517-531.

BHATTACHARYYA, B.K. and K.C. CHAN (1977): Reduction of magnetic and gravity data on an arbitrary surface acquired in a region of high topographic relief, Geophysics, 42, 1411-1430.

BuRG, J.P. (1975): Maximum entropy spectral analysis, PHD Dissertation, Stanford University, UMI Dissertation Service, Ann Arbor Michigan, U.S.A., pp. 168.

CiminAle, M. and M. Loddo (1989): A computer program to perform the upward continuation of potential field data between arbitrary surfaces, Comput. Geosci., 15, 889-903.
Ciminale, M. and M. Loddo (1992): Reply to R.D. Ray and R.O. Hansen's comments on «A computer program to perform the upward continuation of potential field data between arbitrary surfaces», Comput. Geosci., 18, 623.

DAMPNEY, C.N.G. (1969): The equivalent source technique, Geophysics, 34, 39-53.

Eisenstat, S.C., M.H. GuRSKy, M.H. SchultZ and A.H. SHERMAN (1977): Yale sparse matrix package; the nonsymmetric codes, Research Rep. No. 114, Yale University Department of Computer Science, pp. 60.

EMILIA, D.A. (1973): Equivalent sources used as an analytic base for processing total magnetic field profiles, Geophysics, 38, 339-348.

GiBerT, D. and A. GALDEANo (1985): A computer program to perform transformations of gravimetric and aeromagnetic survey, Comput. Geosci., 11, 553-588.

Graber-Brunner, V., E. Klingele and I. Marson (1991): An improved solution for the problem of upward continuation of gravity field data in rugged topography, Boll. Geofis. Teor. Appl., 33, 135-144.

HANSEN, R.O. and Y. MIYAZAKI (1984): Continuation of potential fields between arbitrary surfaces, Geophysics, 49, 787-795.

MendoncA, C.A. and J.B.C. Silva (1994): The equivalent data concept applied to the interpolation of potential field data, Geophysics, 59, 722-732.

NAGY, D. (1966): The gravitational attraction of a right rectangular prism, Geophysics, 31, 362-371.

RAY, R.D. and R.O. HANSEN (1992): Comment on «A computer program to perform the upward continuation of potential field data between arbitrary surfaces» by M. Ciminale and M. Loddo, Comput. Geosci., 15, 889-903, 1989, Comput. Geosci., 18, 619-622.

XIA, J. and D.R. SPRowL (1991): Correction of topographic distortion in gravity data, Geophysics, 56, 537-541. 